\title{
“Atypical” Atypical Parkinsonism: Critical appraisal of a cohort
}

Stephanie T. Hirschbichler, MD, MSc, ${ }^{1}$ Roberto Erro, MD, ${ }^{1,2}$ Christos Ganos, $\mathrm{MD}^{1,4}$ Maria Stamelou, MD, PhD, ${ }^{3}$ Amit Batla, MD, DM, ${ }^{1}$ Bettina Balint, MD ${ }^{1,5}$ Kailash P. Bhatia, MD, FRCP. ${ }^{1}$

${ }^{1}$ Sobell Department of Motor Neuroscience and Movement Disorders, UCL Institute of Neurology, WC1N 3BG London, United Kingdom

${ }^{2}$ Dipartimento di Scienze Neurologiche e del Movimento, Università di Verona, Verona, Italy

${ }^{3}$ Second Department of Neurology, Attiko Hospital, University of Athens, Greece

${ }^{4}$ Department of Neurology, University Medical Center Hamburg-Eppendorf (UKE), Hamburg, Germany

${ }^{5}$ Department of Neurology, University Hospital Heidelberg, Heidelberg, Germany

Classification: full-length article

Correspondence: Kailash P Bhatia, MD, Sobell Department of Motor Neuroscience and Movement Disorders, Institute of Neurology, University College London, Queen Square, London, WC1N 3BG, U.K., E-mail kbhatia@ucl.ac.uk

Running title: "Atypical” Atypical Parkinsonism

Word count: 2998

Number of characters in the title: 57

Number of references/tables/figures: $29 / 3 / 2$

\section{Supplemental figure: 1}

Keywords: atypical parkinsonism, multiple system atrophy, progressive supranuclear palsy, corticobasal degeneration, dementia with lewy bodies, diagnostic research criteria, APphenocopies;

Financial disclosures related to the paper: see below 


\begin{abstract}
Background: Atypical parkinsonian conditions such as multiple system atrophy (MSA), progressive supranuclear palsy (PSP), corticobasal syndrome (CBS) and Dementia with Lewy bodies (DLB) comprise 10-15\% of parkinsonian syndromes. Misdiagnosis with Parkinson disease (PD) and within the entities is common, given the absence of reliable biomarkers. However a correct diagnosis is not only important in clinical practice, but also crucial for any trial attempting to identify biomarkers or new treatments.
\end{abstract}

Methods: Consecutive patients, who were either referred with a diagnosis of a particular AP by a neurologist, or where a movement disorder specialist at Queen Square considered such a diagnosis, were included and the medical records were reviewed retrospectively. We applied each set of current diagnostic research criteria to the respective cohort to see which features fit in and if there are atypical features "outside" the classic definition.

Results: Sixty-nine patients were recruited clinically presenting with one of the following phenotypes: 14 MSA, 24 PSP, 19 CBS and 12 DLB. Up to 49\% showed additional "atypical" features and approximately 10\% eventually received an alternative diagnosis, in half of whom this was based on genetic testing.

Conclusions: In a subset of our patients, despite the final diagnosis of an AP being maintained, there were additional "atypical" features. It remains to be seen if these reflect the clinical heterogeneity of APs, or should prompt a search for an alternative diagnosis. A change in terminology using phenotypic descriptors (e.g. MSA syndrome) rather than aetiological labels, as already applied for CBS, could therefore be a consideration for all the APs. 


\section{Introduction}

Atypical parkinsonism (AP) refers to a group of disorders including multiple system atrophy (MSA), progressive supranuclear palsy (PSP), corticobasal syndrome (CBS) and dementia with Lewy bodies (DLB), which account for up to $10-15 \%$ of all parkinsonian syndromes [1]. Given the lack of diagnostic biomarkers, a definite diagnosis of APs can only be made post mortem [1]. Nonetheless, the classical AP phenotypes have been delineated, and physicians are now familiar with such clinical templates, using them routinely for the diagnosis of these disorders. However, there is increasing evidence that: I) APs can clinically overlap [2,3]; II) some patients with a pathological diagnosis for a certain AP have "atypical" clinical presentations [2-5]; and III) a number of newly discovered genetic conditions might share some clinical features with the "classical APs" (therefore being referred to as "AP-look-alikes") [6]. For example, patients with Dynactin1 (DCTN1) or microtubule-associated protein tau (MAPT) mutations can present with a supranuclear gaze palsy and parkinsonism, as in PSP, although there may be additional features atypical for PSP, and also these cases might be younger at onset than classic PSP. All these factors might contribute to the relatively high rate of misdiagnosis [1], hence hampering the identification of homogenous groups of patients for future research on the pathophysiology of, and putative neuro-protective interventions for, these disorders. Hence in a previous review article we had introduced the term "atypical" atypical parkinsonism to highlight such cases [6]. Here we are reappraising the clinical features and diagnostic outcomes of a large series of AP patients. 


\section{Materials and Methods}

We included consecutive patients referred to us by other neurologists with a working diagnosis of MSA, PSP, CBS, or DLB between January 2013 and May 2015 as a tertiary referral. For each AP patient, clinical details were recorded on a proforma based on current diagnostic research criteria [7-11]. For PSP, two different proformas based on NINDS [10] and NNIPPS [11] sets of criteria were used. Medical records were reviewed retrospectively and the clinical findings were recorded as present or absent during the first examination at our centre. Additional clinical findings (e.g., not mentioned in the research criteria) were also gathered. Finally, imaging findings and results of additional investigations, including genetic testing where available, performed during the diagnostic work-up were also collected. The final diagnosis made at our center was used to classify patients as described in details below.

For the aim of the current study, for each AP patients were allocated into two main groups according to whether or not they fulfilled the research criteria when first attending our clinic (e.g., group A: fulfilling the criteria; and B: not fulfilling the criteria). For each group (e.g. Group A and B), patients were further divided into different subgroups according to the final working diagnosis and the presence of atypical features (either clinical or based on the results of additional investigations) collected throughout a mean follow-up of 1.5 years (range: 6 months-2.5 years). For the aims of the study, we considered features as "atypical", if they are not classically described in a particular AP and were judged too prominent to be "allowed" in that form of AP (for example prominent cognitive dysfunction in MSA). Accordingly, groups A and B were further stratified: group A1), patients fulfilling the criteria of a particular AP and being "typical"; A2), patients having "atypical" features despite fulfilling the criteria for a particular AP; 
A3) patients receiving an alternative diagnosis, when initially fulfilling the criteria for a particular AP; B1) patients not fulfilling the criteria for a particular AP and receiving an alternative diagnosis; and group B2) patients not fulfilling the criteria but still carrying a working diagnosis for that particular AP. A flowchart describing the study design is provided in figure 1.

Given the retrospective nature of the study and the relatively low numbers of patients in each sub-group, statistical analyses were not possible. Written informed consent was obtained from all patients on an ethics committee approved movement disorder research form.

\section{Results}

A total of 69 patients were recruited - 12 with a working diagnosis of DLB, 14 MSA, 24 PSP, and 19 CBS. Table 1 provides demographics and main clinical features of the entire cohort.

\subsection{Dementia with Lewy bodies presentation}

Applying the DLB research criteria, eight patients out of 12 fulfilled the criteria for probable DLB (group A), while the remaining four did not because of the lack of cognitive impairment occurring one year before or after the motor symptom onset (group B).

Among the patients fulfilling the criteria, three had a typical phenotype (group A1), whereas four had additional "atypical" features (table 2), thus falling into group A2. These included two with abnormal eye movements reminiscent of PSP (slow vertical saccades or up-gaze restriction deemed excessive for age) and another two with limb weakness and an associated head-drop, which led us to perform additional genetic analyses. Of the 
latter two, one was found to be heterozygous for the c.1399G>A POLG mutation. The second was detected to have asymmetric atrophy of the hippocampi and given the possible family history of her mother developing cognitive problems in her eighties, genetic testing was pursued. She was found to carry the c.1216C $>\mathrm{T}$ mutation of the MAPT gene, thus leading to a definitive alternative diagnosis, although the criteria for probable DLB were initially fulfilled (group A3).

In group $B$, all patients $(n=4)$ had a final working diagnosis of DLB. (See figure 1A for detailed sub-classification of "DLB-group").

\subsection{Multiple System Atrophy presentation}

Applying the research diagnostic criteria, a total of 12 patients (Group A) fulfilled the criteria, 2 patients for possible and 10 for probable MSA. Of these, 5 had predominant cerebellar features (MSA-C) while 7 had predominant parkinsonian features (MSA-P). Within this group, despite 2 patients having a typical phenotype, the large majority (up to 75\%) had additional "atypical" features (table 2). Namely, two patients had "atypical" eye movements (one with slow vertical saccades and 1 with the "round the houses" sign), three had marked freezing of gait, one visual hallucinations unrelated to drugs, one significant cognitive impairment on neuropsychometry (not fulfilling dementia criteria) and three had a positive family history of dementia (2) and PSP (1). Of interest, among the 10 patients fulfilling the criteria but having atypical clinical features, one MSA-P case had a negative DaTSCAN and another one had an "atypical" MRI (generalised volume loss, signal change in the splenium and thalamus and frontotemporal atrophy). Another patient in this group had the middle cerebellar peduncle (MCP) sign on MRI. Despite having been occasionally reported in MSA, this finding along with progressive cognitive 
decline, led to additional genetic investigations and he was found to be carrier of the FMR1 premutation (90 CGG repeats), thus eventually falling into the Group A3.

Finally, two presenting with a combination of parkinsonism and cerebellar signs, did not fulfil the criteria for MSA (lack of autonomic dysfunction) and they were eventually given a working diagnosis of Parkinsonism-Ataxia Syndrome (group B1). (See figure 1B for detailed sub-classification of "DLB-group").

\subsection{Progressive Supranuclear Palsy presentation}

For the 24 patients within the PSP group, the NINDS and the NNIPPS diagnostic criteria were applied separately. Applying the NINDS criteria, four fitted the criteria for probable and one for possible PSP, whereas 19 patients did not fit the criteria (among whom nine lacked the core criterion of prominent postural instability and falls within the first year from onset and eight had at least one of the exclusion criteria). Applying the NNIPPS criteria, for five patients the diagnosis of PSP was applicable, while within the group not fulfilling the NNIPPS criteria (n=19), 11 did not meet the core criteria and 9 had at least one exclusion criterion, namely rest tremor $(n=4)$, disease duration $>8$ years $(n=3)$ or signs of CBS $(n=6)$. When considering the overlap between the two sets of criteria, only 2 patients fulfilled both, while none of the possible PSP subjects (according to the NINDS) fulfilled the NNIPPS criteria (figure 2).

Subjects were subsequently sub-divided according to whether $(n=8$, group $A)$ or not $(n=$ 16, group B) they fitted either of the two sets of criteria. Among the group A patients, while five patients had a typical phenotype, the other three showed additional "atypical" features (group A2) including pyramidal signs and cerebellar dysfunction (table 3). On 
the other hand, among the patients not fulfilling the criteria $(n=16$; group B), seven $(44 \%)$ received an alternative diagnosis after an extensive work-up (group B1), three of whom carried specific genetic mutations (1 polymerase gamma (POLG), 1 proganulin (PRGN), 1 DCTN1), one was eventually diagnosed with Fahr's disease, and three showed extensive pyramidal signs and marked cognitive impairment suggestive of a frontotemporal dementia with motor neuron disease spectrum. The remaining nine patients were still carrying a working diagnosis of PSP, although not fulfilling the criteria (group B2). Five of them had a clinical phenotype considered typical for PSP (e.g. backward falls and supranuclear gaze palsy (SNGP)), but showed additional CBS-like features that are currently considered an exclusion criterion for the diagnosis according to both sets. In one of the remaining four patients in group B2, post-mortem examination was available confirming the diagnosis of PSP. (See figure 1C for detailed sub-classification of "DLBgroup").

\subsection{Corticobasal Syndrome}

Nineteen patients were included with a referral diagnosis of CBS - 15 had a classic CBS phenotype, one presented with a nonfluent/agrammatic variant of primary progressive aphasia, and three presented with a CBS/PSP-overlap-syndrome (e.g. showing additional vertical supranuclear gaze palsy). Seventeen of them fulfilled current criteria (group A), nine of whom fulfilled possible and 8 probable CBS criteria. Nonetheless, while eight patients had a "typical" phenotype (group A1), seven showed additional "atypical" features (detailed in table 2; group A2). Two eventually received an alternative diagnosis (group A3) since there were no signs of parkinsonism (despite the referral diagnosis of CBS) and there was evidence of marked posterior cortical atrophy on imaging, so that the 
patients were diagnosed with Benson's syndrome (posterior cortical atrophy; atypical variant of Alzheimer disease).

Two patients did not fulfil the criteria (group B): in one a diagnosis of DLB was eventually made (group B1), while in the other the clinical diagnosis remained that of CBS (group B2).

Worth noting, considering all patients for whom the working diagnosis remained that of CBS ( $n=16$; 8 patients in group A1, 7 in group A2, and 1 in group B2; supplemental figure 1D), 13 had a DaTSCAN of which four were normal.

\section{Discussion}

Our results show that the current criteria are too restrictive and "fitting" patients into the existing boxes might lead to misdiagnosis: I) A number of patients carrying a working diagnosis of AP might in fact have a different underlying condition; II) Among the patients for whom the clinical diagnosis of a specific AP would be appropriate according to the research diagnostic criteria, a proportion have additional "atypical" features, which raises the question as to whether they should cast doubt on the diagnosis or, conversely, expand the phenotype of each AP; and III) Some patients with a phenotype suggestive of a specific AP do not fulfil the diagnostic criteria, but might still actually have the condition, which underlines the relatively low sensitivity of the existing criteria used for AP syndromes.

There is increasing evidence that there are some disorders mimicking APs, hence being referred as AP-look-alikes or AP-phenocopies.[6,13-16] This was the case in approximately $5 \%$ our cohort. In some of these patients, there was a definitive genetic diagnosis, indicating the initial working diagnosis was wrong, even though they fulfilled 
the respective research criteria. However, in a proportion of patients with a phenotype suggestive of one of the APs there were some "red flags", including a positive family history (e.g., patients with DCTN1 and MAPT mutations), a "too-long"-standing history (e.g. patient with FMR1 premutation), and associated clinical/radiological features (e.g. severe depression in the patient with DCTN1 mutation; subtle hippocampal atrophy in the patient carrying the MAPT mutation; prominent cognitive impairment and MCP sign in the patient with FMR1 premutation), which prompted us to look for an alternative diagnosis. This would suggest that, despite the core clinical phenotype being suggestive of one of the APs, one should look out for any features that would be "atypical" for the proposed diagnosis. Our results reinforce previous proposals to implement a 4-step approach based on age at onset, tempo of progression, family history and associated clinical features, to improve diagnostic accuracy [6].

Nearly half of our patients fulfilling the criteria for one of the APs showed additional "atypical" features on close scrutiny. While in some cases this led to re-consider the diagnosis, in a substantial proportion of our series the diagnosis could not be revised. It should be remarked that we recorded some clinical features as being atypical on the basis of AP research criteria (e.g., either because they were reported as exclusion criteria or not mentioned at all). However, a body of evidence has been produced that such exclusion criteria can in fact occur in some patients and should not be considered exclusive anymore. For instance, the NNIPPS criteria for PSP consider the presence of a rest tremor as an exclusion criterion for the diagnosis of PSP $[10,11]$, while it is nowadays well known that PSP patients might have rest tremor (PSP-P) [17]. Similarly, both NINDS or the NNIPPS sets of criteria for PSP consider signs of CBS, including alien limb phenomenon, apraxia and severe asymmetric parkinsonism, as exclusion criteria [10,11]. However, a 
recent study on 100 pathological PSP cases has shown that up to 7\% can present with a PSP-CBS overlap phenotype [18].

For other clinical features, it is unclear whether they might occur or not in the context of each AP. For instance, we found that up to $18 \%$ of MSA patients $(2 / 11$ of patients in groups A1 and A2; supplementary figure 1B) showed evidence of cortical involvement (1 with cognitive impairment, on formal neuropsychometry and 1 with visual hallucinations unrelated to medications). Dementia is still considered an exclusion criterion for MSA, but a recent paper discussed that they indeed can have a certain degree of cognitive impairment later in disease course [19]. It remains to be seen how early cognitive impairment can occur in MSA. As for the PSP group, we found that about one third showed cerebellar signs (including limb ataxia, dysmetria and cerebellar eye movements). Cerebellar signs are not classically seen in this condition, but a number of recent reports have provided clinical, imaging and pathological evidence of cerebellar involvement in PSP [20-22]. Once again, future research should clarify if this represents a novel phenotype of PSP (e.g. PSP-C) or not.

Altogether, our results argue for an update of the current criteria that, although originally not meant to be used in routine clinical practice, do not seem to reflect the heterogeneity of each AP. A first attempt has been in fact pursued with corticobasal degeneration (CBD) [9], where: 1) it is advised to use the label CBS rather than CBD; and 2) different criteria exist for CBS diagnosis, profiled according to the different phenotypes presented.

A final comment is also needed on the DaTSCAN results. In a number of patients fulfilling the criteria for a particular AP, DaTSCAN were negative, suggesting they had an alternative diagnosis. However, we did not use this evidence per se to discard the diagnosis, since there is increasing evidence that a minority of patients with degenerative 
parkinsonism, particularly CBS and MSA-C, might in fact have negative scans [23-26]. It remains to be seen whether this is the case in large, pathologically proven, series of APs.

Whilst at one end of the spectrum there were patients fulfilling some AP criteria but proven to have a different condition, at the other end there were patients not fulfilling the research criteria, but being still considered to have that particular AP. One example would be the patient with pathologically proven PSP, who at the time of our first assessment did not fulfil either the NINDS (lack of falls within the first year) or the NNIPPS (lack of supranuclear gaze palsy) criteria. This confirms that such criteria have a relatively low sensitivity, especially early in the disease course [2,27-29]. In our series, this might have been the case in up to $19 \%$. Early identification of these patients is indeed crucial in terms of possible neuroprotective trials and this would reinforce the unmet need for diagnostic biomarkers. Quite obviously, the identification of possible biomarkers for any APs, would be possible only if we are able to select homogenous groups which truly reflect distinct disease entities.

We acknowledge some limitations. First, our study was retrospective, and some clinical features might be unrecorded or overlooked. Moreover, ours is a tertiary referral center for movement disorders, which probably biases referrals more towards "atypical" cases. It may also be the case that some of the "atypical" patients (e.g. groups A2) could actually have other, yet undefined, conditions. However diagnoses were made by clinicians with significant expertise in the field. We classified our series according to the currently available research criteria, some of which are in need of updating, and which were not developed for use in routine clinical settings. However, this seemed to us the only systematic approach to address this topic. Since for a large proportion of our patients a 
conclusive (either genetic or pathological) diagnosis was not available, definitive conclusions cannot be drawn.

Despite these limitations, our preliminary results underpin the clinical and pathological heterogeneity among atypical parkinsonian syndromes. While future, prospective, multicenter studies with confirmatory pathological diagnosis are needed, it would probably be wise, from the clinical standpoint, to start using the AP templates as phenotypic descriptors rather than diagnostic labels. This change in terminology has been already applied to CBS, where we no longer refer to the underlying pathology. Following this line, it might be also useful to start using the concept of typical (and atypical) DLB, MSA, PSP and CBS. Such a pragmatic approach might be useful to identify homogenous groups of patients in whom to search for reliable biomarkers.

\section{Figure caption}

Figure 1. Flowchart describing the study design. A) Clinical data at the first evaluation; B) Clinical and investigation data collected throughout a mean follow-up period of 1.5 years.

Figure 2. Distribution of patients fulfilling either NINDS or/and NNIPPS criteria for the diagnosis of PSP.

Supplementary figure 1 shows the sub-division of the patients for each AP, namely Dementia with Lewy Bodies (1A), Multiple System Atrophy (1B), Progressive Supranuclear Palsy (1C) and Corticobasal Syndrome (1D).

\section{Full financial disclosures}

RE received consultancies from Zambon. 
KPB receives royalties from publication of Oxford Specialist Handbook of Parkinson's Disease and Other Movement Disorders (Oxford University Press, 2008) and of Marsden's Book of Movement Disorders (Oxford University Press, 2012). He received funding for travel from GlaxoSmithKline (GSK), Orion Corporation, Ipsen, and Merz Pharmaceuticals.

All the other authors report no disclosure.

\section{References}

1. Stamelou M, Bhatia KP. Atypical parkinsonism: diagnosis and treatment. Neurol Clin. 2015;33(1):39-56

2. Koga S, Aoki N, Uitti RJ, van Gerpen JA, Cheshire WP, Josephs KA, Wszolek ZK, Langston JW, Dickson DW. When DLB, PD, and PSP masquerade as MSA: An autopsy study of 134 patients. Neurology. 2015;85(5):404-12

3. Batla A, Stamelou M, Mensikova K, Kaiserova M, Tuckova L, Kanovsky P, Quinn N, Bhatia KP. Markedly asymmetric presentation in multiple system atrophy. Parkinsonism Relat Disord. 2013;19(10):901-5.

4. Wenning GK, Tison F, Ben Shlomo Y, Daniel SE, Quinn NP. Multiple system atrophy: a review of 203 pathologically proven cases. Mov Disord 1997;12:133-147.

5. Josephs KA, Petersen RC, Knopman DS, et al. Clinicopathologic analysis of frontotemporal and corticobasal degenerations and PSP. Neurology 2006;66:41-48

6. Stamelou M, Quinn NP, Bhatia KP. "Atypical” atypical parkinsonism: New genetic conditions presenting with features of progressive supranuclear palsy, corticobasal degeneration, or multiple system atrophy-A diagnostic guide. Mov. Disord. 2013;28(9):1184-1199.

7. McKeith IG, Dickson DW, Lowe J, et al. Diagnosis and management of dementia with Lewy bodies: third report of the DLB Consortium. Neurology. 2005;65(12):1863-72.

8. Gilman S, Wenning GK, Low PA, et al. Second consensus statement on the diagnosis of multiple system atrophy. Neurology 2008;71:670-676.

9. Armstrong MJ, Litvan I, Lang AE, et al. Criteria for the diagnosis of corticobasal degeneration. Neurology. 2013;80(5):496-503.

10. Litvan I, Agid Y, Calne D, et al. Clinical research criteria for the diagnosis of progressive supranuclear palsy (Steele-Richardson-Olszewski syndrome): report of the NINDS-SPSP international workshop. Neurology. 1996;47(1):1-9

11. Bensimon G, Ludolph A, Agid Y, Vidailhet M, Payan C, Leigh PN; NNIPPS Study Group. Riluzole treatment, survival and diagnostic criteria in Parkinson plus disorders: the NNIPPS study. Brain. 2009 Jan;132(Pt 1):156-71 
12. Neeve VC, Samuels DC, Bindoff LA, et al. What is influencing the phenotype of the common homozygous polymerase- $\gamma$ mutation p.Ala467Thr? Brain. 2012;135(Pt 12):3614-26.

13. Tremolizzo L, Bertola F, Casati G, Piperno A, Ferrarese C, Appollonio I. Progressive supranuclear palsy-like phenotype caused by progranulin p.Thr272fs mutation. Mov Disord 2011;26:1964-1966.

14. Lindquist $S$, Duno $M$, Batbayli $M$, et al. Corticobasal and ataxia syndromes widen the spectrum of C90RF72 hexanucleotide expansion disease. Clin Genet 2013;83:279-283

15. Erro R, Lees AJ, Moccia M, Picillo M, Penco S, Mosca L, Vitale C, Barone P. Progressive parkinsonism, balance difficulties, and supranuclear gaze palsy. JAMA Neurol.

2014;71(1):104-7

16. Newsway V, Fish M, Rohrer JD, et al. Perry syndrome due to the DCTN1 G71R mutation: a distinctive levodopa responsive disorder with behavioral syndrome, vertical gaze palsy, and respiratory failure. Mov Disord 2010;25:767-770

17. Williams DR, de Silva R, Paviour DC, Pittman A, Watt HC, Kilford L, Holton JL, Revesz T, Lees AJ. Characteristics of two distinct clinical phenotypes in pathologically proven progressive supranuclear palsy: Richardson's syndrome and PSP-parkinsonism. Brain. 2005;128(Pt 6):1247-58

18. Respondek G, Stamelou M, Kurz C, et al. The phenotypic spectrum of progressive supranuclear palsy: a retrospective multicenter study of 100 definite cases. Mov. Disord. 2014;29(14):1758-66.

19. Stankovic I, Krismer F, Jesic A, et al. Cognitive impairment in multiple system atrophy: a position statement by the Neuropsychology Task Force of the MDS Multiple System Atrophy (MODIMSA) study group. Mov Disord. 2014;29(7):857-67

20. Cerebellar involvement in progressive supranuclear palsy: A clinicopathological study. Kanazawa M, Shimohata T, Toyoshima Y, Tada M, Kakita A, Morita T, Ozawa T, Takahashi H, Nishizawa M. Mov Disord. 2009;24(9):1312-8

21. Sawa N, Kataoka H, Kiriyama T, Izumi T, Taoka T, Kichikawa K, Ueno S. Cerebellar dentate nucleus in progressive supranuclear palsy. Clin Neurol Neurosurg. 2014;118:326.

22. Iwasaki Y, Mori K, Ito M, Tatsumi S, Mimuro M, Yoshida M. An autopsied case of progressive supranuclear palsy presenting with cerebellar ataxia and severe cerebellar involvement. Neuropathology. 2013;33(5):561-7.

23. McKinley J, O'Connell M, Farrell M, et al. Normal dopamine transporter imaging does not exclude multiple system atrophy. Parkinsonism Relat Disord 2014;20:933-4.

24. O’Sullivan SS, Burn DJ, Holton JL, et al. Normal dopamine transporter single photonemission CT scan in corticobasal degeneration. Mov Disord 2008;23:2424-6.

25. Kaasinen V, Gardberg M, Röyttä M, et al. Normal dopamine transporter SPECT in neuropathologically confirmed corticobasal degeneration. J Neurol 2013;260:1410-11. 
26. Erro R, Schneider SA, Stamelou M, Quinn NP, Bhatia KP. What do patients with scans without evidence of dopaminergic deficit (SWEDD) have? New evidence and continuing controversies. J Neurol Neurosurg Psychiatry. 2015 May 19. doi: 10.1136/jnnp-2014310256. [Epub ahead of print]

27. Respondek G, Roeber S, Kretzschmar H, et al. Accuracy of the National Institute for Neurological Disorders and Stroke/Society for Progressive Supranuclear Palsy and neuroprotection and natural history in Parkinson plus syndromes criteria for the diagnosis of progressive supranuclear palsy. Mov Disord. 2013;28(4):504-9

28. Doran M, Du Plessis DG, Enevoldson TP, Fletcher N a., Ghadiali E, Larner a. J. Pathological heterogeneity of clinically diagnosed corticobasal degeneration. J Neurol Sci 2003;216(1):127-134.

29. Weerkamp NJ, Tissingh G, Poels PJ, Zuidema SU, Munneke M, Koopmans RT, Bloem BR. Diagnostic accuracy of Parkinson's disease and atypical parkinsonism in nursing homes. Parkinsonism Relat Disord. 2014;20(11):1157-60 